\title{
Communication
}

\section{A Phononic Crystal-Based High Frequency Rheometer}

\author{
Maxime Lanoy ${ }^{1}$, Alice Bretagne ${ }^{2}$, Valentin Leroy ${ }^{3}$ and Arnaud Tourin ${ }^{2, *}$ \\ 1 Department of Physics and Astronomy, University of Manitoba, Winnipeg, MB R3T 2N2, Canada; \\ maxime.lanoy@umanitoba.ca \\ 2 ESPCI Paris, PSL University, CNRS, Institut Langevin, 75005 Paris, France; arnaud.tourin@espci.fr \\ 3 CNRS, Laboratoire Matière et Systèmes Complexes, Université Paris Diderot, 75013 Paris, France; \\ valentin.leroy@univ-paris-diderot.fr \\ * Correspondence: arnaud.tourin@espci.fr; Tel.: +33-180-963-063
}

Received: 16 March 2018; Accepted: 29 April 2018; Published: 1 May 2018

\begin{abstract}
Dynamic Mechanical Analysis (DMA) allows for the measurement of the complex shear modulus of an elastomer. Measurements at frequencies above the frequency range of the device can be reached thanks to the Time-Temperature Equivalence principle. Yet, frequencies higher than a few $\mathrm{kHz}$ are not attainable. Here, we propose a method exploiting the physics of bubble phononic crystals to measure the complex shear modulus at frequencies of a few tens of $\mathrm{kHz}$. The idea is to fabricate a phononic crystal by creating a period arrangement of bubbles in the elastomer of interest, here PolyDiMethylSiloxane (PDMS), and to measure its transmission against frequency. Fitting the results with an analytic model provides both the loss and storage moduli. Physically, the shear storage modulus drives the position of the dip observed in transmission while the loss modulus controls the damping, and thus the level of transmission. Using this method, we are able to compare the high-frequency rheological properties of two commercial PDMS and to monitor the ageing process.
\end{abstract}

Keywords: acoustics; phononic crystals; metamaterials; rheology; shear storage modulus; shear loss modulus

\section{Introduction}

A series of recent works have shown that phononic crystals, or metamaterials, based on bubbles trapped in a soft elastic matrix can exhibit exotic properties such as negative refraction [1], superabsorption [2], or subwavelength focusing [3]. In all these works, the key is that a spherical air cavity in an elastic matrix is an oscillator, much like a bob on a spring, which resonates at a natural frequency given by

$$
\omega_{0}=1 / a \sqrt{\left(3 \beta_{g}+4 G^{\prime}\right) / \rho}
$$

with $\beta_{g}$ the bulk modulus of the gas inside the bubble, $\rho$ and $G^{\prime}$ the mass density and shear storage modulus of the host elastic matrix, respectively, and $a$ the air cavity radius. This formula tells us that the restoring force is due to both the elasticity of the gas and the shear elasticity of the solid while the inertia is that of the solid. Provided that the matrix is soft enough, the resonance is associated to a wavelength much larger than the air cavity size as is the case for the so-called Minnaert frequency resonance of an air bubble in a liquid (i.e., with $G^{\prime}=0$ ).

Based on periodic arrangements of air cavities trapped in PolyDiMethylSiloxane (PDMS), metascreens with adjustable transmission properties can be realized [4,5]. A simple analytic model proposed in [6] allows the transmission and reflection of such metascreens to be predicted. In this short communication, we show reciprocally that this model can be used to infer the high-frequency rheological properties of cross-linked soft polymers in which bubbles are trapped with a controlled 
periodicity. We demonstrate that this kind of "phononic crystal-based rheometer" allows for high frequency measurements, which would be not attainable with classical rheometers.

\section{Materials and Methods}

The behavior of viscoelastic materials can be described by their complex shear modulus, $G(\omega)=$ $G^{\prime}(\omega)+i G^{\prime \prime}(\omega)$, defined as the ratio of the shear stress to the shear strain. The storage modulus, $G^{\prime}$, represents the in-phase (elastic) stress response, while the shear loss modulus, $G^{\prime \prime}$, is related to the dynamic viscosity $\eta$ through $G^{\prime \prime}(\omega)=\eta \omega$. Dynamic mechanical analysis (DMA) is a standard technique for measuring the frequency, temperature dependence, or both, of both moduli. For example, Figure 1 shows a typical variation of both the storage and the loss modulus of a PDMS sample measured at $20{ }^{\circ} \mathrm{C}$. It was obtained with an imposed deformation of $5 \%$ with frequencies varying between $0.01 \mathrm{~Hz}$ and $20 \mathrm{~Hz}$.

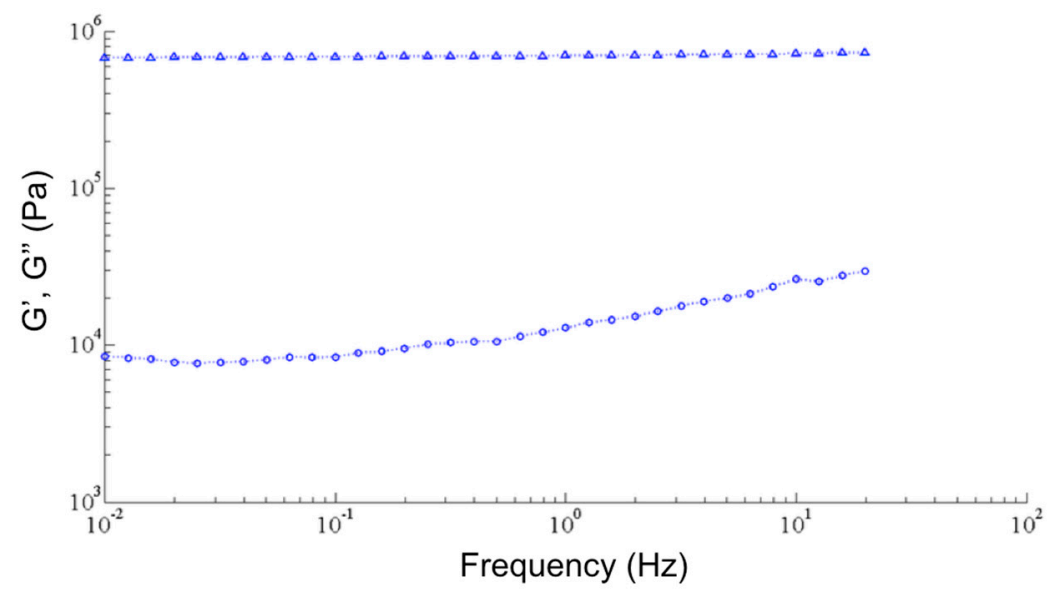

Figure 1. Typical variation of storage $(\Delta)$ and loss (o) moduli of PolyDiMethylSiloxane (PDMS) at $20{ }^{\circ} \mathrm{C}$.

By repeating the same kind of measurement at lower temperatures, the behavior of PDMS at higher frequencies can be inferred beyond the frequency range of the apparatus. The idea is to use the principle of Time-Temperature Equivalence (TTE) to generate a master curve of storage modulus vs. frequency at a particular temperature. The TTE principle states that the change in temperature from the measurement temperature, $T$, to the reference, $T_{0}$, is equivalent to multiplying the frequency scale by the so-called horizontal translation factor $a_{T}\left(T, T_{0}\right)$. For example, Palade et al. [7] were able to determine the viscoelastic properties of polybutadienes over 15 decades of frequencies window by performing rheological tests over a temperature range of $200{ }^{\circ} \mathrm{C}$. However, for some polymers the application of the TTE is limited to a temperature range fixed by the crystallization temperature ( $-40{ }^{\circ} \mathrm{C}$ for PDMS). Moreover, for most polymers, the translation factor takes different values for different relaxation (terminal or segmental) modes [8,9], thus making the TTE difficult to apply.

Here, we propose an alternative method to perform high frequency measurements. The idea is to form a one-layer crystal of bubbles by trapping air cavities in the elastomer of interest (here PDMS), to measure its acoustic transmission and to adjust the results by the simple analytic model presented in [6]. The one-layer crystal of bubbles is fabricated using soft lithography techniques (Figure 2). One first creates a mold consisting of a two-dimensional square arrangement of cubes. The cube side length is $a_{\text {cube }}=590 \mu \mathrm{m}$ and the lattice constant is $b=2 \mathrm{~mm}$. PDMS is soft enough such that the cube resonance is associated to a wavelength much larger than the air cavity size and pretty much the same as the one of a sphere with the same volume $[4,10,11]$. The radius of the equivalent sphere is given by $a=\sqrt[3]{\frac{3}{4 \pi}} a_{\text {cube }}$. A thin layer of PDMS is then deposited on the mold and cured at $65^{\circ} \mathrm{C}$ during $45 \mathrm{~min}$. We thus obtain a patterned PDMS layer that we close by a pure PDMS layer. 
(a)

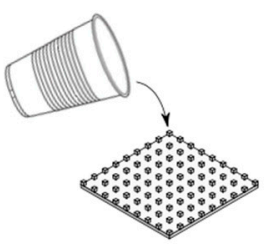

(b)

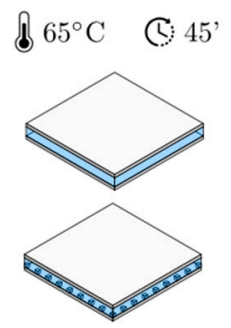

(c)

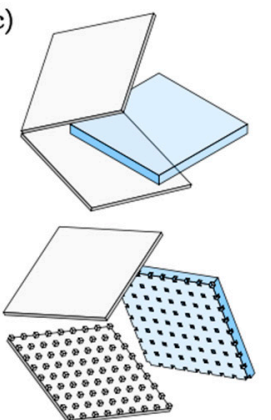

(d)

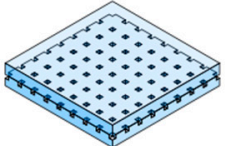

Figure 2. Practical realization of a monolayer bubble crystal in PDMS: (a) The monomer-crosslinking mixture is poured onto a mold; (b) After sealing, the sample is cured for $45 \mathrm{~min}$ at $65^{\circ} \mathrm{C}$. The operation is repeated with a second, non-textured mold; (c) After curing, the polymer becomes solid and can be unmolded; (d) The two blocks of PDMS are joined so that the bubbles are isolated from the outside.

Then, the transmission coefficient of such a one-layer crystal is predicted to be [6]:

$$
t=1+\frac{i K a}{\left(\frac{\omega_{0}^{2}}{\omega^{2}}-1+2 \sqrt{\pi} \frac{a}{b}\right)-i(\delta+K a)}
$$

where $\delta$ is the dissipative damping constant of the bubbles, and $K a=\left(\frac{\lambda}{b}\right)^{2} \frac{k a}{2 \pi}$ with $\lambda$ the wavelength for longitudinal waves in PDMS.

For a gas cavity in a soft elastic medium dissipative damping is dominated by viscous losses such that $\delta=4 \eta /\left(\rho a^{2} \omega\right)$ where $\eta$ and $\rho$ are the viscosity and density of PDMS, respectively [12]. $\eta$ is assumed to be independent of frequency in the frequency range of interest. $K a$ is a super-radiative term telling us that the bubble layer radiates $N / 2 \pi$ times more than one single bubble, where $N=\left(\frac{\lambda}{b}\right)^{2}$ represents the number of bubbles that oscillate in phase. We would like to stress that Equation (2) incorporates all multiple scattering events in the bubble layer and that it predicts a minimum in transmission at $\omega_{0} / \sqrt{(1-2 \sqrt{\pi} a / b)}$.

Transmission measurements were carried out in a large water tank (Figure 3). A piezoelectric transducer generates a short pulse that propagates through water, traverses the bubble crystal, and reaches the hydrophone. To mimic a one-dimensional configuration, the sample is placed in the far field of the transducer. Edge effects were overcome by mounting the sample on the aperture $(5 \mathrm{~cm}$ diameter) of an acoustically opaque screen.

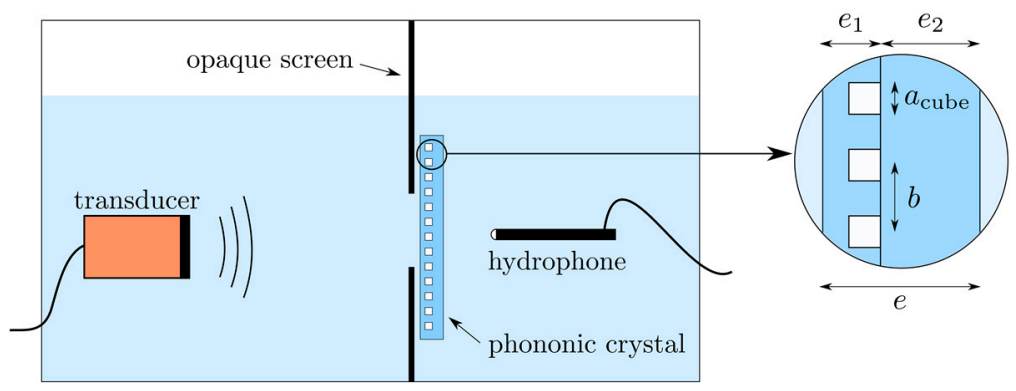

Figure 3. Setup for acoustic transmission measurements. A short ultrasonic pulse is emitted from the transducer and the transmission is probed using a hydrophone. Sample parameters are $a_{\text {cube }}=$ $590 \mu \mathrm{m}, b=2 \mathrm{~mm}, e_{1}=0.8 \mathrm{~mm}, e_{2}=5 \mathrm{~mm}$. 


\section{Results}

We studied two different commercial PDMS: Sylgard 184 and RTV615. The composition of these two products is carefully kept secret, but the RTV615 is known to be softer than Sylgard 184 and thus more convenient for the fabrication of microvalves in microfluidic devices. We have tested these two materials with our method. Following the process described in the previous section, we have fabricated two bubble crystals with the same structural parameters and measured their transmission coefficients (Figure 4).

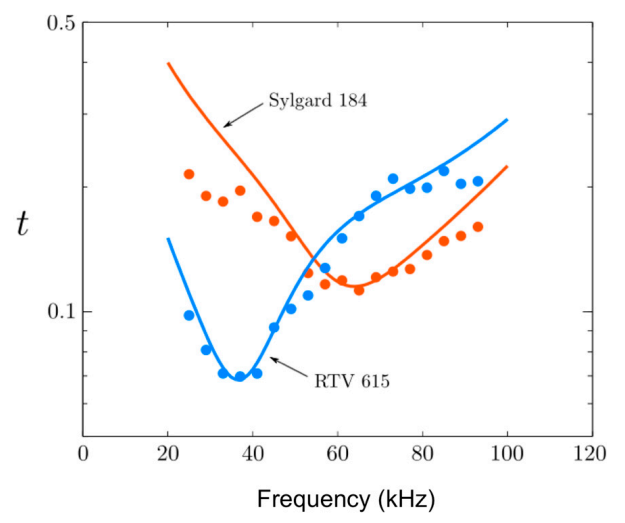

Figure 4. Transmission coefficients. Measurements (full circles) are adjusted by Equation (2) (full lines).

We would like to point out that the gap observed in Figure 4 does not originate from Bragg scattering but rather from hybridization between the free space propagation mode and the bubble Minnaert resonance $[13,14]$. Such a gap is known to survive disorder $[15,16]$. The position of the transmission dip is driven by the storage modulus while its height is related to the loss modulus. The inferred values are listed in Table 1. The results confirm that RTV615 is softer and less viscous than Sylgard, which explains why it is preferably used for the fabrication of microvalves.

Table 1. Values of storage modulus and viscosity for two kinds of PDMS.

\begin{tabular}{ccc}
\hline PDMS & $G^{\prime}$ (MPa) & $\eta$ (Pa.s) \\
RTV615 & $0.60 \pm 0.05$ & $1.5 \pm 0.1$ \\
Sylgard & $1.8 \pm 0.1$ & $2.5 \pm 0.2$ \\
\hline
\end{tabular}

Note that the uncertainty measurement originates from the margin of error in the cavity size (of the order of $10 \mu \mathrm{m}$ ). Given these promising results, we decided to use our method to monitor the ageing of PDMS, a phenomenon the users of PDMS are familiar with. Although appreciated for its great versatility, this material causes difficulties in terms of repeatability. The chain crosslinking mechanism is a complex phenomenon that depends on working conditions and is often incomplete. A very simplified image consists in picturing the polymer as a complex entanglement of chains with a degree of cross-linking which increases during the polymerization. As a consequence, it quickly becomes difficult for free chains to reach the reactive sites. A well-known manifestation of this phenomenon is the evolution of the interfacial properties of PDMS. While being naturally hydrophobic, PDMS is often treated with oxygen plasma to obtain good conditions of wetting. However, this treatment does not resist in the long-term because of the migration of low molecular weight chains to the surface. However, if these chains move under the effect of diffusion, they are also required to continue crosslinking according to very specific time scales.

To illustrate this phenomenon, we have performed time-lapse measurements of the acoustic transmission through a periodic layer of bubbles embedded in RTV615. Figure 5 shows the acoustic transmission curves obtained respectively $1 \mathrm{~h}, 1$ day, and 9 days after curing. Ageing manifests itself in the shift of the resonance towards higher frequency, which reflects an increase in the storage modulus. 


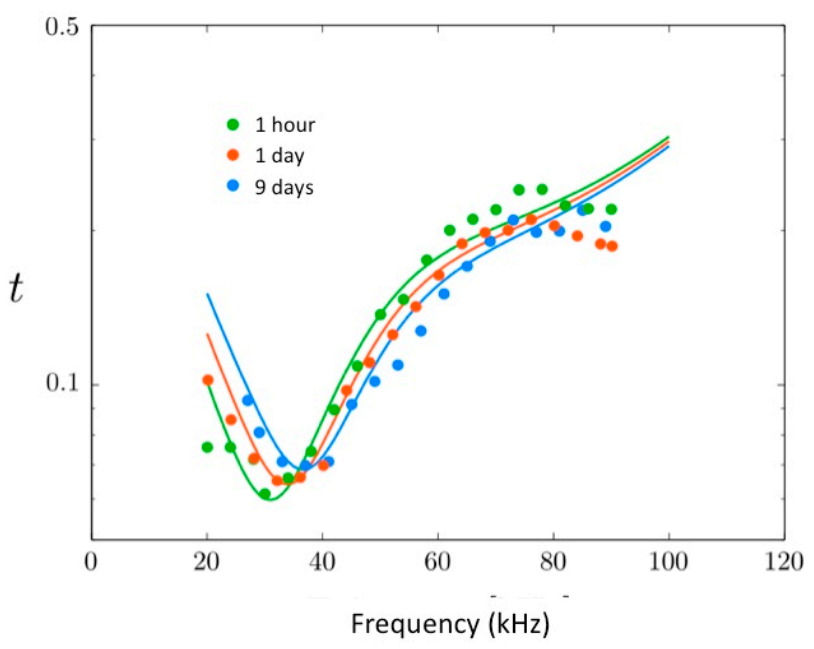

Figure 5. Transmission through the bubble phononic crystal at different times reveals ageing of PDMS. Measurements (full circles) are adjusted by Equation (2) (full lines).

This result is consistent: the higher the degree of crosslinking, the less chain rearrangements are likely to occur. The response to a solicitation then becomes essentially elastic and PDMS hardens. From a more quantitative point of view, we obtained a good agreement with the model for the values listed in Table 2.

Table 2. Evolution in time of storage modulus and viscosity for PDMS RTV615.

\begin{tabular}{ccc}
\hline Time & $G^{\prime}(\mathbf{M P a})$ & $\eta$ (Pa.s) \\
\hline $1 \mathrm{~h}$ & $0.4 \pm 0.05$ & $1.4 \pm 0.01$ \\
1 day & $0.5 \pm 0.05$ & $1.4 \pm 0.01$ \\
9 days & $0.6 \pm 0.05$ & $1.5 \pm 0.01$ \\
\hline
\end{tabular}

\section{Discussion}

For some polymers, the crystallization phenomenon limits the temperature range which can be explored such that the determination of the shear modulus remains limited to low frequencies. Several strategies based on the measurement of both the velocity and the attenuation of shear waves have been proposed in the past for reaching higher frequencies up to the $\mathrm{MHz}$ range. Reflection measurements are preferably used due to the strong attenuation of shear waves in viscoelastic materials at these frequencies. For example, in [17] the authors exploit the echoes reflected at the interface between the sample of interest and a piece of quartz, the latter being used as a delay line. The echoes measured in absence of the sample (i.e., at the quartz/air interface) are taken as a reference. The storage and loss shear modulus can then be determined from the $\mathrm{n}^{\mathrm{e}}$ echo by taking the ratio of its amplitude to the reference and the difference between its arrival time and the one of the reference (Figure 6).

The method however requires frequencies sufficiently high such that the successive echoes do not overlap. The length of the delay line can be increased to overcome this difficulty, the price to pay being diffraction effects that blur the results. Furthermore, a good coupling between the viscoelastic material and the delay line is crucial.

Our alternative approach avoids these difficulties. We have shown that bubbles trapped in a viscoelastic material to form a bubble phononic crystal can be used to probe its rheological properties in a frequency domain (a few tens of $\mathrm{kHz}$ ) hard to reach with either DMA or acoustic reflection measurements. Of course, some further tests will be required to fully validate this approach as a new rheology measurement technique. Such a method should be useful to probe the rheological 
properties of cross-linked polymers in an intermediate frequency range (i.e., from a few tens of $\mathrm{kHz}$ up to a few $\mathrm{MHz}$ ) not accessible by common techniques. Interestingly, it appears as an unexpected outcome of the work we have done for the last ten years on the exotic transmission properties of bubble phononic crystals.

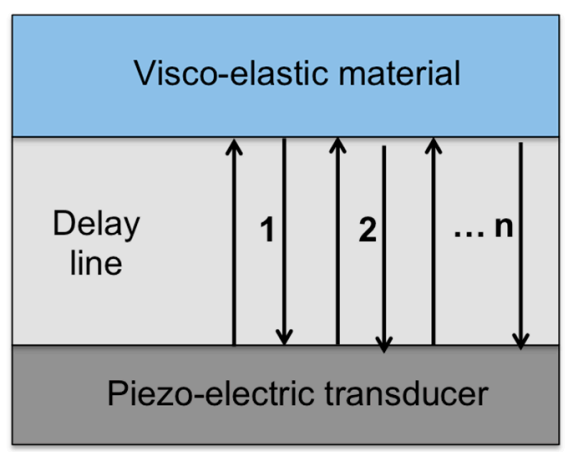

Figure 6. Acoustic measurement of the complex shear modulus of a viscoelastic material. A short pulse is sent from a shear wave piezo-electric transducer through a delay line. The storage and loss moduli can be deduced from the amplitude and arrival time of the $n^{e}$ echo compared to the reference.

Author Contributions: M.L., V.L., and A.T. conceived and designed the experiments; A.B. and M.L. performed the experiments and analyzed the data; A.T. wrote the paper.

Acknowledgments: This work was supported by LABEX WIFI (Laboratory of Excellence ANR-10-LABX-24) within the French Program "Investments for the Future" under Reference No. ANR-10-IDEX-0001-02 PSL*. We thank Helene Montes (SIMM, ESPCI Paris) for DMA measurements.

Conflicts of Interest: The authors declare no conflict of interest. The founding sponsors had no role in the design of the study; in the collection, analyses, or interpretation of data; in the writing of the manuscript; and in the decision to publish the results.

\section{References}

1. Lanoy, M.; Page, J.H.; Lerosey, G.; Lemoult, F.; Tourin, A.; Leroy, V. Acoustic double negativity induced by position correlations within a disordered set of monopolar resonators. Phys. Rev. B 2017, 96, 220201. [CrossRef]

2. Leroy, V.; Strybulevych, A.; Lanoy, M.; Lemoult, F.; Tourin, A.; Page, J.H. Super-Absorption of Acoustic Waves with Bubble Meta-Screens. Phys. Rev. B 2015, 91, 020301. [CrossRef]

3. Lanoy, M.; Pierrat, R.; Lemoult, F.; Fink, M.; Leroy, V.; Tourin, A. Subwavelength focusing in bubbly media using broadband time reversal. Phys. Rev. B 2015, 91, 224202. [CrossRef]

4. Leroy, V.; Bretagne, A.; Fink, M.; Willaime, H.; Tabeling, P.; Tourin, A. Design and Characterization of bubble phononic crystals. App. Phys. Lett. 2009, 95, 171904. [CrossRef]

5. Bretagne, A.; Tourin, A.; Leroy, V. Enhanced and reduced transmission of acoustic waves with bubble meta-screens. App. Phys. Lett. 2011, 99, 221906. [CrossRef]

6. Leroy, V.; Strybulevich, A.; Scanlon, M.G.; Page, J.H. Transmission of ultrasound through a single layer of bubbles. Eur. Phys. J. E 2009, 29, 123-130. [CrossRef] [PubMed]

7. Palade, L.I.; Verney, V.; Attane, P. Time-temperature superposition and linear viscoelasticity of polybutadiennes. Macromolecules 1997, 30, 5897-5904.

8. Roland, C.M.; Ngai, K.L.; Satangelo, P.G.; Qiu, X.H.; Ediger, M.D.; Plazek, D.J. Temperature Dependence of Segmental and Terminal Relaxation in Atactic Polypropylene Melts. Macromolecules 2001, 34, 6159-6160. [CrossRef]

9. Plazek, D.J.; Ngai, K.L. Correlation of polymer segmental chain dynamics with temperature-dependent time-scale shifts. Macromolecules 1991, 24, 1222-1224. [CrossRef]

10. Meyer, E.; Brendel, K.; Tamm, K. Pulsation oscillations of cavities in rubber. J. Acoust. Soc. Am. 1958, 30, 1116-1124. [CrossRef] 
11. Calvo, D.C.; Thangawng, A.L.; Layman, C.N. Low-frequency resonance of an oblate spheroidal cavity in a soft elastic medium. J. Acoust. Soc. Am. 2012, 132, EL1-EL7. [CrossRef] [PubMed]

12. Prosperetti, A. Thermal effects and damping mechanisms in the forced radial oscillations of gas bubbles in liquids. J. Acoust. Soc. Am. 1977, 61, 17-27. [CrossRef]

13. Kushwaha, M.S.; Djafari-Rouhani, B.; Dobrzynski, L. Sound isolation from cubic arrays of air bubbles in water. Phys. Lett. A 1998, 248, 252-256. [CrossRef]

14. Sainidou, R.; Stefanou, N.; Modinos, A. Linear chain of weakly coupled defects in a three-dimensional phononic crystal: A model acoustic waveguide. Phys. Rev. B 2006, 74, 172302. [CrossRef]

15. Kafesaki, M.; Penciu, R.S.; Economou, E.N. Air Bubbles in Water: A Strongly Multiple Scattering Medium for Acoustic Waves. Phys. Rev. Lett. 2000, 84, 6050-6053. [CrossRef] [PubMed]

16. Leroy, V.; Bretagne, A.; Lanoy, M.; Tourin, A. Band gaps in bubble phononic crystals. AIP Adv. 2016, 6, 121604. [CrossRef]

17. Gasparoux, J. Etude Rhéo-Acoustique de Gels Polymers Polydiméthylsiloxanes. Ph.D. Thesis, Université Montpellier 2, Montpellier, France, 2007.

(C) 2018 by the authors. Licensee MDPI, Basel, Switzerland. This article is an open access article distributed under the terms and conditions of the Creative Commons Attribution (CC BY) license (http://creativecommons.org/licenses/by/4.0/). 